\title{
Thyroid abscess as a complication of percutaneous ethanol ablation of cystic thyroid nodules
}

\author{
Francesk Mulita ${ }^{1}$, Levan Tchabashvili' ${ }^{1}$, Georgios-Ioannis Verras ${ }^{1}$, Elias Liolis ${ }^{2}$, Stamatia Siouti ${ }^{3}$, \\ Konstantinos Panagopoulos ${ }^{1}$, Michail Vailas ${ }^{1}$
}

${ }^{1}$ Department of Surgery, General University Hospital of Patras, Patras, Greece

${ }^{2}$ Department of Internal Medicine, Division of Oncology, General University Hospital of Patras, Patras, Greece

${ }^{3}$ Faculty of Emergency Nursing, General University Hospital of Patras, Patras, Greece

Key words: thyroid abscess; ethanol ablation; thyroidectomy; thyroid nodule

Thyroid nodule is a very common disorder in clinical practice. According to recent guidelines, image-guided nonsurgical techniques such as ethanol and thermal ablation have been proposed as alternative and minimally invasive procedures for the treatment of benign symptomatic thyroid nodules [1-2].

A 63-year-old male with significant past medical history of multinodular goitre and diabetes type 2 underwent US-guided percutaneous ethanol ablation (PEA) because of a 6-cm cystic nodule in the right lobe of the thyroid. The procedure was well tolerated by the patient, and no complications were noted. A week after the procedure, he was admitted to our clinic with fever and a painful right-sided neck swelling for 48 hours. Apart from pyrexia at $38.2^{\circ} \mathrm{C}$, his vital signs were normal. On examination he was found to have a right-sided thyroid swelling that was warm and tender, but no cervical lymphadenopathy was present.

Laboratory control revealed slightly elevated levels of C-reactive protein (CRP) as well as a mild leucocytosis. Ultrasonography (US) was performed and revealed a cystic lesion in the right lobe with approximately $80 \mathrm{~mL}$ of heterogenous content. Under real-time US guidance, a 21-gauge needle connected to a $20-\mathrm{mL}$ syringe was inserted into the lesion, and $30 \mathrm{~mL}$ of liquefied pus was aspirated. The day after abscess aspiration, the patient's pain disappeared, and his body temperature normalized. Repeat aspiration was not performed. The microbiological culture revealed a ciprofloxacin-susceptible Staphylococcus aureus infection, and an antibiotic therapy with ciprofloxacin was therefore prescribed. The patient received intravenous ciprofloxacin and amikacin for 10 days during his hospitalization and was discharged in good clinical condition. However, he was readmitted 6 days after the discharge because of high-grade pyrexia at $39.1^{\circ} \mathrm{C}$. On examination, the patient's vital signs were unremarkable. He was symptom free and the inflammatory markers (WBC, CRP) were within the normal range. A computed tomography (CT) scan of the neck was performed, which confirmed the abscess in the right thyroid lobe. There was tracheal deviation to the left, and an abscess cavity $4.5 \times 3 \times 2.5 \mathrm{~cm}$ in size. In addition, signs of gas collection could be seen (Fig. 1). The patient underwent subtotal thyroidectomy. He did not have any postoperative complications and was discharged on the 6th postoperative day in good clinical condition. The surgical specimen of thyroid was received for histological examination and was suggestive of acute suppurative thyroiditis.

Thyroid abscess formation, while itself rare, is the most common short-term consequence of acute suppurative thyroiditis (AST), an uncommon occurrence, with an estimated incidence $\sim 0.5 \%$ of all thyroid disease. Both these entities are most often encountered in children, women aged 20-40 years, and patients with pre-existing thyroid conditions, namely, nodular goitre, thyroid cancer, or Hashimoto's disease or immunocompromised patients, especially AIDS patients [3]. It is largely accepted that the thyroid gland exhibits a high degree of innate immunity from bacterial colonization, attributed to the gland's thick fibrous capsule, rich blood supply, high iodine concentrations, and abundant and multi-directional lymphatic drainage. The anatomic origin of AST and thyroid abscess formation is thoroughly understood, with the presence of a pyriform sinus fistula being the leading cause of bacterial translocation, 


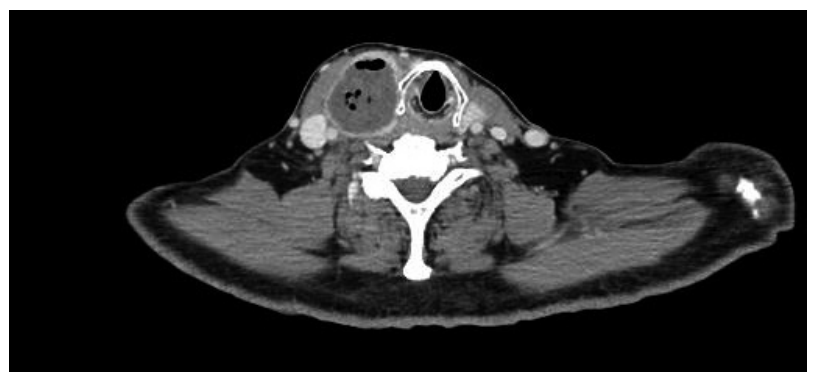

Figure 1. CT scan of the neck showed the abscess in the right thyroid lobe. Tracheal deviation to the left, and an abscess cavity, $4.5 \times 3 \times 2.5 \mathrm{~cm}$. Signs of gas collection could be seen

especially among children. Other well-known routes of infection are a direct tissue spread of neck abscess, haematogenous or lymphatic spread, and neck trauma. Infrequent causes of thyroid infection such as fistulizing oesophageal carcinoma, foreign-body ingestion with subsequent oesophageal perforation or septic emboli due to infective endocarditis, as well as a form of idiopathic thyroid abscess have also been described [3-4].

Laboratory parameters are commonly non-specific and include the following: elevated WBC (between 10,000 and 21,000 as described in a case series), neutrophilia, elevated ESR, and elevated CRP. Blood and urine cultures are routinely obtained, and an antibiotic resistance panel is requested [5]. The most helpful imaging method in an acutely presenting thyroid abscess is ultrasound. Sonographic study helps differentiate an abscess from solid and cystic masses, as well as from other forms of thyroiditis, and it helps to guide the FNA biopsy, the surgical evacuation, or topical antibiotic administration. The use of neck CT scan is reserved for secondary evaluation, to visualize the relationship of the abscess with adjacent neck structures. MRI, radionuclide imaging, laryngoscopy, barium swallow, and upper GI endoscopy are reserved for difficult differentials $[3,5]$.

Treatment of a diagnosed thyroid abscess should be prompt, due to its lethal potential. While exact and definitive guidelines do not yet exist, it is common practice to start with immediate administration of broad-spectrum antibiotics. Common regimens, include the following: piperacillin-tazobactam, cefazolin, cefotaxime, meropenem, with the adage of vancomycin on suspected MRSA involvement [6]. Thyroid abscess with airway compromise has an absolute indication for either immediate needle or open surgical drainage. Surgery is also indicated in persistent thyroid abscess, despite adequate antibiotic administration, or clinical deterioration. The procedures utilized are either abscess drainage and removal or total, near total, or partial thyroidectomy, with resection of thyroid tissue being elected for extensive necrotic or infracted areas of the gland [3]. A major surgical consideration to be factored in is the avoidance of surgery during the acute phase of the abscess formation, because it has been shown to increase adverse outcomes and morbidity. Surgical intervention is also needed for the definitive management of anatomic defects that are causal of the abscess (pyriform sinus fistula), for which endoscopic obliteration or fibrin glue application is also an option [6].

\section{Informed consent}

Informed consent was obtained from the patient for the publication of this article.

\section{Conflict of interest}

None declared.

\section{Funding sources}

None declared.

\section{Authors' contributions}

All authors contributed equally to the article.

\section{References}

1. Hahn SY, Shin JH, Na DG, et al. Korean Society of Thyroid Radiology (KSThR), Korean Society of Radiology. Ethanol Ablation of the Thyroid Nodules: 2018 Consensus Statement by the Korean Society of Thyroid Radiology. Korean J Radiol. 2019; 20(4): 609-620, doi: 10.3348/kjr.2018.0696, indexed in Pubmed: 30887743.

2. Gharib H, Papini E, Garber JR, et al. AACE/ACE/AME Task Force on Thyroid Nodules. American Association of Clinical Endocrinologists, American College Of Endocrinology, And Associazione Medici Endocrinologi medical guidelines for clinical practice for the diagnosis and management of thyroid nodules - 2016 update. Endocr Pract. 2016; 22(5): 622-639, doi: 10.4158/EP161208.GL, indexed in Pubmed: 27167915

3. Pearce EN, Farwell AP, Braverman LE. Thyroiditis. [published correction appears in N Engl J Med. 2003 Aug 7;349(6):620]. N Engl J Med. 2003; 348(26): 2646-2655, doi: 10.1056/NEJMra021194, indexed in Pubmed: 12826640 .

4. Cawich SO, Hassranah D, Naraynsingh V. Idiopathic thyroid abscess. Int J Surg Case Rep. 2014; 5(8): 484-486, doi: 10.1016/j.ijscr.2014.05.019, indexed in Pubmed: 24981167.

5. Paes JE, Burman KD, Cohen J, et al. Acute bacterial suppurative thyroiditis: a clinical review and expert opinion. Thyroid. 2010; 20(3): 247-255, doi: 10.1089/thy.2008.0146, indexed in Pubmed: 20144025.

6. Yedla N, Pirela D, Manzano A, et al. Thyroid Abscess: Challenges in Diagnosis and Management. J Investig Med High Impact Case Rep. 2018; 6: 2324709618778709, doi: 10.1177/2324709618778709, indexed in Pubmed: 29854858. 